\title{
A rare malignancy in a patient with neurofibromatosis: gastric adenocarcinoma
}

\author{
Bora Barut, ${ }^{1}$ Volkan Ince, ${ }^{1}$ Fatih Ozdemir,${ }^{1}$ Murat Harputluoglu, ${ }^{2}$ Burak Isik ${ }^{1}$ \\ ${ }^{1}$ Department of General Surgery, Inonu University Faculty of Medicine, Malatya, Turkey \\ ${ }^{2}$ Department of Gastroenterolgy, Inonu University Faculty of Medicine, Malatya, Turkey
}

\begin{abstract}
Neurofibromatosis is an autosomal dominant disease that is characterized by cutaneous hyperpigmentation and multiple neurofibromas and often accompanied by localized peripheral and central neural tumors. Gastrointestinal involvement is seen in $25 \%$ of the cases. Gastrointestinal stromal tumors, pancreatic and duodenal neuroendocrine tumors are the most common tumors in patients with neurofibromatosis. Neurofibromatosis and gastric adenocarcinoma constitute an extremely rare combination. In this case report we aim to present a patient with neurofibromatosis operated due to gastric adenocarcinoma.
\end{abstract}

Keywords: Adenocarcinoma; neurofibromatosis; stomach.

$\mathrm{N}$ eurofibromatosis type 1 (von Recklinghausen disease) is an autosomal dominant disease that is characterized by cutaneous hyperpigmentation and multiple neurofibromas and often accompanied by localized peripheral and central neural tumors. The most frequently seen symptoms are café-au-lait spots, cutaneous neurofibroma, peripheral or central nervous system tumors [1]. In $25 \%$ of the patients with neurofibromatosis gastrointestinal involvement is observed. The most frequently seen tumors associated with neurofibromatosis are gastrointestinal stromal tumors, neurofibroma, and carcinoid tumors [2]. Very rarely it can be associated with carcinomas of esophagus, stomach, duodenum, small intestine, and hepatobiliary system [3].

\section{CASE REPORT}

In a 67-year-old male patient, upper gastrointestinal endoscopy performed upon complaint of the patient revealed a polypoid mass extending from gastric corpus up to antrum which was histopathologically diagnosed upon examination of the biopsy specimen excised from this mass lesion. With caféau-lait spots detected all over his body (Figure 1A), and neurofibromas (Figure 1B) the patient was evaluated in collaboration with the department of dermatology, and the diagnosis of neurofibromatosis was made. On examinations performed by Departments of Eye Diseases, and Neurosurgery, any involvement of eye, and brain could not be detect- 

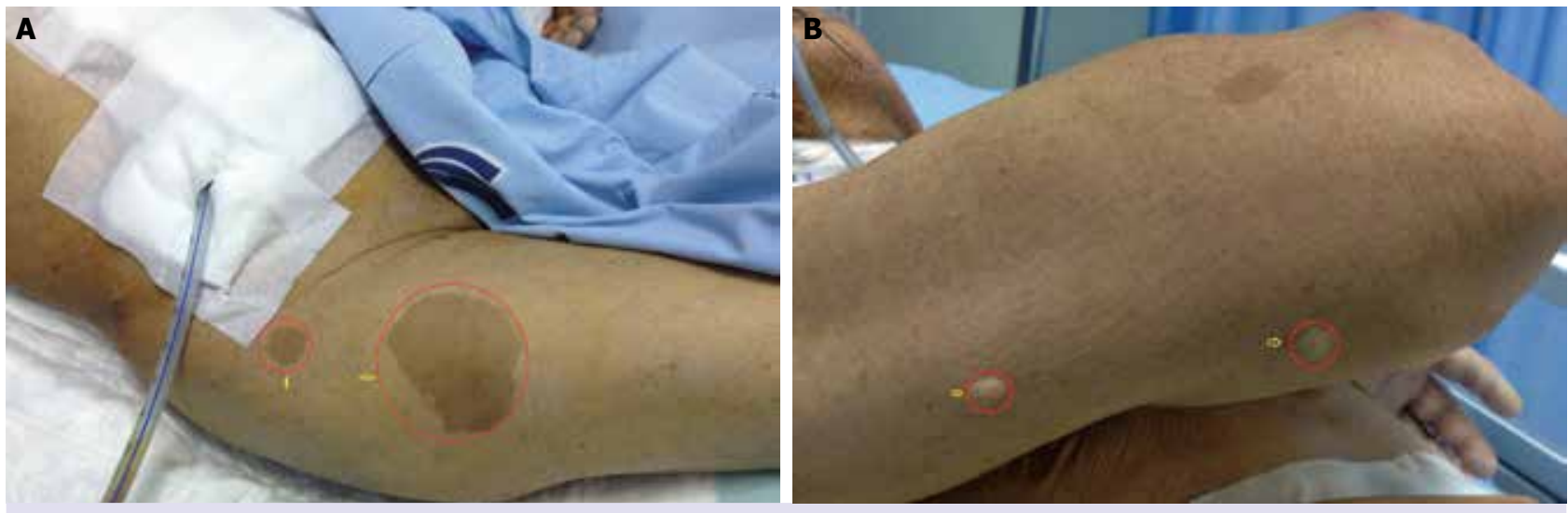

FiguRE 1. (A) Café-au-lait spots. (B) Neurofibromas.

ed. On abdominal computed-tomograms, a $6 \mathrm{~cm}$ polypoid mass localized on the greater curvature of the stomach, and extending up to the lumen, and a nearly $1 \mathrm{~cm}$-long hypodense lesion in the $8^{\text {th }}$ hepatic segment were observed (Figure 2). This lesion on the 8 . hepatic segment was rather evaluated by radiologists as a simple cyst. Therefore, any preoperative intervention targeted at this lesion was not considered, and perioperative excisional biopsy was decided on.

At admission, his whole blood counts, and biochemical parameters were within normal limits. Only CA 19-9 level was increased $(48.3 \mathrm{U} / \mathrm{ml}$ [normal 0-33 U/ml]).
During laparotomy, a mass lesion extending beyond gastric serosa, and multiple intraabdominal lymph nodes along the hepatodudodenal ligament, and in the paraaortic space were observed. Besides on the omentum, and peritoneum tumoral implants were detected. Lesion on the 8 . hepatic segment was excised, and sent for frozen section examination which was reported as a benign lesion. For palliation, total gastrectomy, and Roux-N-Y esophagojejunostomy were performed. Any postoperative complication was not observed. Postoperative histopathology was reported as moderately differentiated adenocarcinoma, and multiple metastatic nodes. The patient was discharged on the 7 . day with cure.

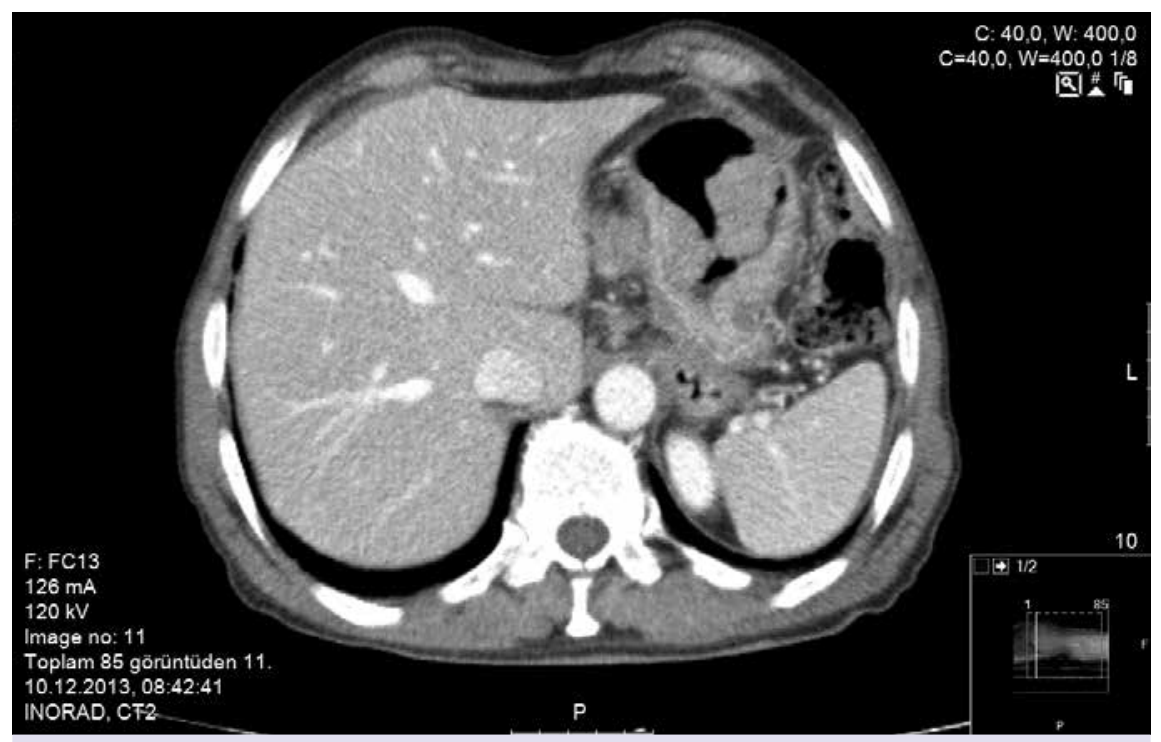

FIGURE 2. Computed tomography of the patient. 
During postoperative period the patient is currently being followed up by the departments of eye diseases, and neurosurgery regarding the presence of neurofibromatosis. Department of dermatology initiated medical treatment. In addition, eye, and brain involvement were not encountered in examinations, and tests. The patient is being followed up without detection of any problem.

\section{DISCUSSION}

Neurofibromatosis Type I (von Recklinghausen disease) was firstly diagnosed in the year 1882. It is an autosomal dominant genetic disease seen in every 3000 births which develops secondary to mutation on the chromosome 17q11.12. In this disease newly formed mutations are frequently seen with an equal distribution between male, and female patients $[1,4]$.

In association with neurofibromatosis, neurogenic, and endocrine tumors as meningioma, glioma, pheochromacytoma can be seen in $3-5 \%$ of the cases [5]. In a few cases concomitancy between neurofibromatosis, and gastric adenocarcinoma has been reported [6]. In neurofibromatosis patients with gastrointestinal tumors signs, and symptoms as abdominal pain, bleeding, and manifestations of intussusception are present, and most of the cases are incidentally detected during laparotomies performed for other indications, postmortem examinations, and gastrointestinal barium series [7]. In patients with neurofibromatosis the most frequently seen gastrointestinal tumors are stromal tumors which frequently localized on stomach, and small bowels [4].

\section{Conclusion}

Since gastrointestinal tumors in patients with neurofibromatosis do not yield many specific symp- toms, diagnosis of this disease is usually delayed. In fact, our patient who had not any complaint other than hematemesis, the diagnosis of gastric adenocarcinoma could be made only following endoscopic examinations. To prevent delays in diagnosis, patients with the established diagnosis of neurofibromatosis should be examined as for the presence of gastrointestinal malignancies, and apart from gastrointestinal stromal tumors, diagnosis of gastric adenocarcinoma should be kept in mind.

Conflict of Interest: No conflict of interest was declared by the authors.

Financial Disclosure: The authors declared that this study has received no financial support.

\section{REFERENCES}

1. Kato K, Nagase A, Onodera K, Matsuda M, Iwasaki Y, Kato Y, et al. Primary adenocarcinoma of the stomach in von Recklinghausen's disease with high serum levels of multiple tumor markers: a case report. J Med Case Rep 2011;5:521.

2. Goldman H, Antonioli DA. Mucosal biopsy of the esophagus, stomach, and proximal duodenum. Hum Pathol 1982;13:42348.

3. Verma M, Loughrey MB. Hepatoid gastric adenocarcinoma in a patient with type 1 neurofibromatosis. Histopathology 2011;58:799-801.

4. Seymour-Dempsey K, Andrassy RJ. Neurofibromatosis: implications for the general surgeon. J Am Coll Surg 2002;195:55363.

5. Davis GB, Berk RN. Intestinal neurofibromas in von Recklinghausen's disease. Am J Gastroenterol 1973;60:410-4.

6. Basu S, Majumdar J, Mitra R, Chowdhury JR. Primary adenocarcinoma of the stomach associated with peripheral neurofibromatosis: report of a case. Surg Today 1997;27:57-9.

7. Stratopoulos C, Papakonstantinou A, Anagnostopoulos G, Terzis I, Tzimas G, Gourgiotis S, et al. Intestinal neurofibromatosis and small-bowel adenocarcinoma: a single case study. Eur J Cancer Care (Engl) 2009;18:466-9. 